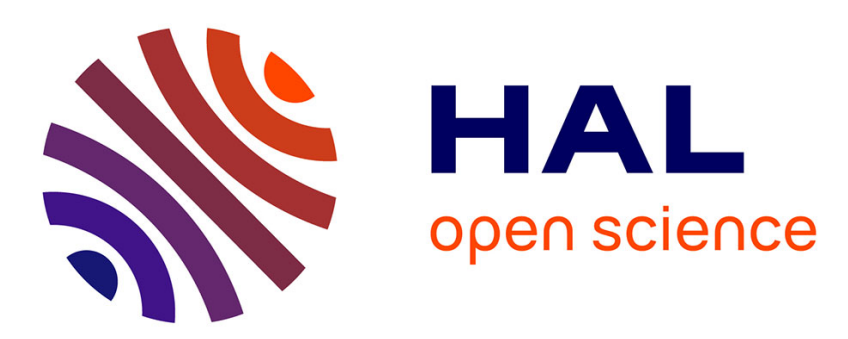

\title{
Attention, observation et notation en situation de groupe dans une perspective psychanalytique \\ Guy Gimenez
}

\section{To cite this version:}

Guy Gimenez. Attention, observation et notation en situation de groupe dans une perspective psychanalytique. Revue de psychothérapie Psychanalytique de Groupes, 2015, L'observation psychanalytique dans les pratiques groupales, 63, pp.79-94. hal-01338402

\section{HAL Id: hal-01338402 \\ https://hal-amu.archives-ouvertes.fr/hal-01338402}

Submitted on 30 Jun 2016

HAL is a multi-disciplinary open access archive for the deposit and dissemination of scientific research documents, whether they are published or not. The documents may come from teaching and research institutions in France or abroad, or from public or private research centers.
L'archive ouverte pluridisciplinaire HAL, est destinée au dépôt et à la diffusion de documents scientifiques de niveau recherche, publiés ou non, émanant des établissements d'enseignement et de recherche français ou étrangers, des laboratoires publics ou privés. 
L'observation psychanalytique en situation de groupe pose la question des niveaux de réalités psychiques que nous souhaitons repérer et des modalités de fonctionnements psychiques adéquats pour rendre possible ce repérage. Le projet de ce travail est de faire le point sur les qualités d'une méthode d'observation psychanalytique pertinente pour saisir ces niveaux de réalités psychiques spécifiques et d'étudier les conditions rendant possible une telle activité.

Le travail d'observation est antérieur à celui de la prise de notes (Gimenez, barthélémy, 2011) ainsi que de la notation interne. Il en est le soubassement et la condition. L'observation dont il sera question ici sera pluri-vertex, polytopique, et plurifocale : pluri-vertex (prenant en compte et intégrant plusieurs points de vue et positions psychiques du clinicien : comme l'ouverture et la défocalisation) ; polytopique (portant sur plusieurs lieux psychiques : sur ce qui se passe hors de l'observateur, dans l'observateur et dans le lien (clinicien-cothérapeute, clinicien-groupe) et entre l'observateur et le groupe); et plurifocale (assumant le fait qu'elle implique plusieurs modalités de distance à l'objet-groupe : proximité en étant totalement dans ce qui se passe icimaintenant et distance minimale autoréflexive impliquée par la position méta, auto-observateur de ce qui se passe, y compris de l'activité d'observation elle-même).

L'OBSERVATION DE «CE QUI EST » SENSORIEL : (ECOUTE DES FAITS)

Le premier niveau d'observation correspond au premier niveau de réalité psychique du groupe : le perceptuel. Quand nous conduisons 
un groupe, nous faisons une expérience plurisensorielle et perceptive complexe : de nous-mêmes, des autres, du groupe. L'attention est ici tournée vers le dehors. Dans la prise de notes, ces éléments observés sont consignés dans la première colonne. Nos organes des sens captent une partie de ce que bion (1970) nomme " les faits de la séance » qui correspondent à « ce qui est », qui se présente à notre appareil perceptif. Sur le fond plus ou moins silencieux du psychisme, vont se découper des formes, appréhendées comme des percepts initialement (d'abord) non saturés par la signification (et le sens) et qui vont devoir trouver place puis être organisés dans le monde interne du clinicien. Cette discrimination entre éléments pertinents et non pertinents et leur différenciation $\mathrm{du}$ fond silencieux seront vectorisées par l'attention, mais aussi, nous le verrons plus loin, par nos théories explicites et implicites qui en déterminent le champ d'action.

\section{Sélection des canaux sensoriels}

De façon classique, certains canaux sensoriels sont surinvestis par rapport à d'autres comme l'auditif et le visuel : notre attention porte en grande partie sur les discours, le sonore, la rythmicité, la gestuelle, les mimiques, les mouvements, les attitudes. L'olfactif, le cénesthésique et le gustatif peuvent être ponctuellement utilisés. Dans certains groupes traversant des moments régressifs importants, les autres sens peuvent être investis de façon spécifique : l'olfactif peut par exemple prendre une place importante, comme nous avons pu le voir dans le travail avec les groupes dos à dos.

\section{Saturation par la sensorialité}

Quand on commence à observer des groupes, on est frappé par la quantité " d'informations sensorielles » qui parviennent à notre conscience. On peut, dans certains cas, vivre une impression de sursaturation. Nous ne parvenons pas à repérer, saisir, traiter les données trop nombreuses, arrivant de façon brute, non organisée, simultanée, comme une cacophonie dans laquelle nous nous perdons. On peut ainsi se sentir dans certains groupes (par exemple les groupes de sujets psychotiques) submergé par le matériel clinique apparaissant chaotique et confusionnant, dans une sorte de «babélisation »: comme si, dans le groupe, chacun parlait sa propre langue, vivait dans son monde, sans traducteur.

\section{Saturation par la sensorialité dans un groupe transculturel}

Une expérience d'observation et de conduite de groupe dans le cadre de la Société européenne d'Analyse Transculturelle de Groupes (aetga), à Marsala, m'a permis d'explorer cette babélisation hors 
groupes de psychotiques. Elle est pour moi une illustration de la butée de l'observation du matériel clinique sensoriel. Elle nous permettra de faire le lien avec les autres niveaux de réalité psychique à observer. L'aetga, fondée au début des années 1980, a pour but de mettre en place les conditions d'une approche psychanalytique des principes organisateurs de l'identité culturelle, pour les comprendre et les trai-ter. Les séminaires psychanalytiques de groupe dit « transculturels » se tiennent dans une ville et un pays chaque année différents et sont conduits par des psychanalystes de cultures nationales, religieuses et linguistiques différentes et de sensibilités psychanalytiques diverses. $\mathrm{Y}$ alternent des groupes médians (une trentaine de participants), et des groupes larges, réunissant l'ensemble des groupes médians, et pouvant aller jusqu'à cent cinquante personnes. Tous les participants sont des praticiens du groupe originaire de différents pays, la grande majorité de la communauté européenne. Les groupes médians, formés de personnes pouvant parler des langues différentes, sont conduits par deux psychanalystes. Ces derniers garantissent les règles du groupe.

Les séances de groupe large sont conduites par un petit groupe de psychanalyste de cultures psychanalytiques et de langues différentes. Aucun dispositif de traduction n'est prévu. Chacun est invité à exprimer ce qui lui vient (libre association), dans la langue qu'il souhaite, en s'abstenant de toute autre modalité substitutive d'expression et de relation. Je devais initialement, à Marsala, conduire un groupe composé d'une majorité de participants français. Une collègue italienne, de formation foulkienne, également psychanalyste de groupe, et qui parlait un peu le français et l'anglais devait en être coconductrice. Finalement, aucun français ne s'étant inscrit, je me retrouvais dans un groupe de participants italiens, espagnols et allemands pour la plupart, trois langues que je ne comprenais pas. Je commence le groupe avec une grande appréhension. Les participants parlent dans un premier temps essentiellement en italien. Quelques-uns, très rares, prennent la parole en anglais. Contre-transférentiellement, je repère à l'intérieur de moi une grande inquiétude de passer à côté de la chaîne associative groupale, de ne pas comprendre ce qui se dit, de ne pas bien accompagner le groupe en cas de difficulté ou de tension...

Je tends l'oreille, je me focalise sur les mots, et plus je m'applique à le faire, plus j'entre dans une immense confusion, accompagnée d'une sensation de ne pas être à ma place, de ne pas être compétent. Je prends le risque de parler, en français, alors que je sais que pratiquement personne ne me comprendra. Et je sens bien que je suis à côté : à côté de moi-même, à côté du groupe et de sa chaîne associative, à côté de ma coconductrice du groupe. Je me sens perdu, comme dans un pays étranger sans traducteur. Incompris et incompréhensible. Je me sens très décalé par rapport à ma collègue. Je me dis que nous ne parviendrons pas à conduire le groupe ensemble, je me demande ce que je fais là. Au bout d'un moment, j'arrête de lutter et je me focalise 
sur la musique des mots, sur le rythme des phrases en italien. Je me mets à rêver ce qui se dit dans le groupe. Et dans cette rêverie, qui devient douce et agréable, me revient un souvenir d'enfance : celui de moments privilégiés, où ma grand-mère me racontait des histoires en espagnol, langue que je ne connaissais pas. Je retrouve alors, en souve-nir, la douceur de sa voix rassurante et contenante. Je me souviens aussi comment le petit garçon que j'étais se focalisait très attentivement sur la musique de mots, le rythme des phrases, l'émotionnalité rythmique, et les mimiques de son visage buriné. Alors qu'une autre partie de moi continue à écouter ce qui se passe dans le groupe, je m'étaye sur ces souvenirs pour me recentrer et je me rends compte que je commence à lâcher prise...

Je me laisse alors glisser, au-delà de la compréhension et de la signification, dans l'enveloppe sonore du groupe, porté par la musique des mots et le bercement de la rythmicité émotionnelle du groupe. Je me sens, enfin, ici et maintenant, dans le groupe, à ma place. J'habite le moment présent. Et je prends la parole, quand les mots se forment dans ma bouche. Je réponds, ainsi en écho, à ce qui vient d'être dit dans une langue que je ne connais pas. Je parle alors au groupe, en français, de ce que je ressens, de ce qui se passe pour moi dans le groupe et ce que je repère de la tonalité des échanges, de leur vivacité, du ton grave qui est employé, des regards intenses partagés. Je parle du fait que j'ai eu besoin de temps pour intégrer le groupe, et que maintenant je me sens à ma place avec eux, pour les accompagner. Je fonctionne alors au-delà et en deçà des mots : au niveau de l'affect, de l'émotion, et de mes réactions contre-transférentielles et intertransférentielles. À la pause, ma collègue italienne qui conduisait le groupe avec moi me dit l'inquiétude qu'elle avait eue en écho à la mienne alors que mes pre-mières interventions, un peu tendues, étaient complètement à côté de ce qui se passait dans le groupe. Elle me fait part de comment, après une heure, ce que je disais était en phase et proche, et proche ce qu'elle aurait pu dire ou souhaité dire...

\section{L'OBSERVATION DES MOUVEMENTS INTERNES DU CLINICIEN : PENSEES, AFFECTS, EMOTIONS, CONTRE-TRANSFERT ET INTERTRANSFERT}

Cet exemple clinique nous permet d'introduire le second niveau d'observation, qui correspond au second niveau de réalité psychique du groupe : les mouvements internes du clinicien, qu'il s'agisse des pensées, des émotions et des affects. L'attention est ici tournée vers le dedans. Dans la prise de notes, ces éléments internes observés sont consignés dans la seconde colonne, venant compléter la première. Quand nous conduisons un groupe, en écho à notre expérience perceptive, notre appareil psychique produit des pensées, des émotions et des affects. Nous pensons que ce matériel clinique subjectif - les mouvements internes du clinicien - doit être repéré et mis en lien avec 
le matériel sensoriel qu'il peut informer, enrichir et compléter, comme écho de la relation aux membres du groupe, (Freud, 1910 ; Searles, 1979).

\section{Auto-observation dans un groupe à l'aetga}

Dans l'exemple du groupe de l'aetga, je peux auto-observer en moi, la première heure, une grande appréhension, un sentiment d'incompétence, la sensation de ne pas être à ma place, le sentiment d'être perdu et désorienté, incompris et incompréhensible. Ces mouvements émotionnels contre-transférentiels se figurent en moi dans des scénarios de dévalorisation et d'incompétence. Ils s'expriment ensuite également dans la dynamique du lien intertransférentiel (Kaës, 1976, 1982), c'està-dire dans le lien avec ma coconductrice du groupe. J'ai l'impression de ne pas être à la hauteur vis-à-vis d'elle dans le dispositif mis en place, et je crains de ne pouvoir fonctionner avec elle. Ma collègue me raconte, après la première séance, que la chaîne associative qui s'est développée est celle-ci : " On veut faire un grand voyage », " On pense aller très loin ", et "Après un long périple, on se rend compte qu'on est revenu au pays d'origine ». La question du groupe étant de savoir si on accepte d'aller dans un lieu vraiment étranger, avec des personnes vraiment différentes de soi. J'étais alors ému de voir combien les scénarios que j'avais vécus contre-transférentiellement était en partie des figurations de ce qui se passait dans ce groupe, à mon insu : "Quelqu'un se perd au bout du monde pour se retrouver dans son pays d'origine »; "Quelqu'un (un membre du groupe, un enfant) écoute sans comprendre (l'autre, un groupe) "; " On (une grand-mère, des membres d'un groupe) raconte des histoires à quelqu'un (un clinicien, un enfant) qui ne comprend pas les mots $»$; une personne (un enfant, un clinicien) accepte de lâcher le connu pour se mettre en phase rythmique avec de l'étranger inquiétant, et retrouve le lien avec l'autre (avec le groupe).

\section{Un état de réceptivité}

Portons ici quelques instants notre attention sur l'état psychique du (ou des) clinicien qui détermine le " degré d'ouverture » et de réceptivité de l'observation. Nous faisons l'hypothèse que l'observation peut être rendue plus précise par une attitude attentivement flottante et non saturée, ouverte à la surprise et à l'inattendu. L'attention, pour nous, est un ingrédient important de l'observation. elle a été étudiée par Freud, et bion (1970) après lui. Nous proposons de la définir momentanément comme le «pré-investissement de ce qui n'est pas encore arrivé, dit ou fait dans la séance : elle permet de repérer des indices cliniques qui, sinon, seraient passés inaperçus ॥. L'attitude attentive peut être ouverte à l'inconnu (à la relation d'inconnu, dirait Rosolato), et donc à ce qui 
est étranger au dedans. Pour cela, elle doit être détendue, relâchée, défocalisée. C'est dans ce sens que Freud a travaillé sur l'attention flottante (ou plus précisément « également flottante ») pour rendre compte d'une modalité spécifique de fonctionnement du clinicien permettant de recevoir, repérer et traiter les productions d'associations libres du patient, parce que tolérant la surprise, l'inattendu et l'inconnu et les manifestations de l'inconscient. W.R. bion (1970) invite les cliniciens à approfondir l'attitude de «l'attention flottante » telle que la présente $\mathrm{S}$. Freud. Dans cette démarche, et pour être dans un état de réceptivité

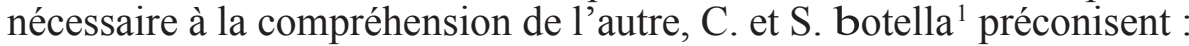
"L'abandon chez l'analyste des représentations-but », comme Freud le suggère, et l'acceptation, " au-delà de l'attention flottante ", d'un " état singulier de passivité du moi, de relâchement, d'ouverture des mailles du réseau d'investissement », ce qui permet le développement de l'intuition, qui, comme le rappelle bion ${ }^{2}$ est un terme qui vient du latin [intueri] qui signifie : voir [tueri] dedans [in].

C. et S. botella explorent cette voie ouverte par bion en termes de « régression formelle de la pensée » : ils ne s'intéressent pas à une technique ou une méthode, mais au contraire à « des surgissements inattendus d'un état régressif de la pensée de l'analyste, à l'insu de son moi et le surprenant à chaque fois ${ }^{3} \gg$. Cet état de régression formelle de la pensée peut être utilisé pour repérer, puis traiter du matériel clinique qui, autrement, aurait pu passer inaperçu : la régression formelle, en tant qu'état psychique régressif, effet du ratage, de la faille ou de l'absence des instances régulatrices et frénatrices de $1^{\prime}$ hallucinatoire ${ }^{4}$, fait émerger chez le clinicien une capacité à « percevoir une intelligibilité qui ne fait pas intervenir les processus secondaires et qui donne accès à certaines zones psychiques autrement inatteignables ${ }^{5} »$. La régression formelle de la pensée est ainsi présentée comme un des moyens d'approche de la "non-représentation », ce qu'on appelle fréquemment le «négatif».

Bion $^{6}$ va encore plus loin et émet l'hypothèse que le clinicien peut parvenir à une réceptivité qui peut lui permettre, "en se dépouillant du souvenir et du désir "», de se mettre en état d'unisson (at-one-ment ${ }^{7}$ ) avec le groupe et d'intuitionner ${ }^{8}$ la réalité psychique des individus et du groupe. Cet état nous semble proche de l'état méditatif ou contem-platif de certains mystiques, qui permet au mental de demeurer paisible

1. C. et S. botella, « La problématique de la régression formelle de la pensée et de l'hallucinatoire », Revue française de psychanalyse, 1989, p. 68.

2. W.R. bion, L'attention et l'interprétation (1970), Paris, Payot, 1974, p. 30 et 33.

3. C. et S. botella, « La problématique de la régression formelle de la pensée et de l'hallucinatoire », op. cit., p. 67-68.

4. Ibid., p. 82.

5. Ibid., p. 73.

6. W.R. bion, L'attention et l'interprétation, op. cit., p. 75.

7. Ibid., p. 76. 
et silencieux. Les sujets se placent dans un état centré sur le moment présent, dans lequel les souvenirs, les pensées, les désirs, les croyances demeurent en suspens. Le mode de fonctionnement psychique habituel nommé « duel » laisse la place à un fonctionnement dit «non-duel » : pouvant être situé en amont de la pensée, de la représentation et des concepts. La démarche consiste à remonter en " amont », au moment de la perception et de l'effet qu'elle produit en soi, avant le travail du psychisme qui " saisit », modifie, traite, transforme de l'étranger et de l'inassimilable en assimilable et en compatible avec le monde du dedans.

Ce que bion (1970) décrit comme « transformation dans $\mathrm{O} »$ semble se rapprocher de la description de ces états d'être. Cette position psychique est rendue possible, chez le clinicien, grâce à une certaine discipline et une certaine vigilance, lui permettant d'être " sans mémoire ni désir ». Bion décrit l'effet (régressif) produit par la rencontre entre deux ou plus de deux appareils psychiques et les transformations qui peuvent en résulter. Il appelle « transformation dans $\mathrm{O} » 1$ 'ensemble de ces transformations sur le clinicien. Le psychisme du clinicien étant transformé par " l'état d'être » de l'interlocuteur ou du groupe. La transformation qui s'effectue dans la psyché du clinicien peut amener à de courts moments de dépersonnalisation, ou d'expériences de vide, d'angoisses sidérantes. Le clinicien peut alors faire l'expérience, pas-sagère, du néant, du non-sens, du trou noir, tel que le décrit Grotstein (1981) en écho aux angoisses très primitives, expérience encore nom-mée agony par Winnicott (1974). En situation de groupe, la «trans-formation dans $\mathrm{O} »$ renvoie à une réactivation des groupes internes du ou des cliniciens, qu'il s'agisse des groupes internes archaïques ou élaborés, et amène le(s) clinicien(s) à être en contact non avec une seule voix, mais avec une polyphonie (écoute et observation polyphonique) ou dans certains cas une cacophonie... (babélisation).

\section{LE REPERAGE DES SCENARIOS DE BASE ET LEUR SUCCESSION : LA CHAINE ASSOCIATIVE GROUPALE}

Le troisième niveau d'observation correspond au troisième niveau de réalité psychique du groupe : il s'agit du repérage des scénarios de base et de leur succession. Quand nous conduisons un groupe, nous faisons l'expérience d'une production plurielle de discours, produits par les participants. L'attention est ici focalisée sur le discours de chacun, sur l'interdiscursivité, et ce qui en constitue le fondement. Dans la prise de notes, ces éléments observés sont consignés dans la troisième colonne. En situation de groupe, les discours s'organisent entre eux en formant ce que René Kaës a nommé une " chaîne associative groupale » et qui est un " discours tenu à plusieurs voix ». L'écouter nécessite d'être attentif à « la polyphonie de l'intersubjectivité (ce qui 
se passe entre les sujets du groupe) et de l'interdiscursivité ${ }^{\prime \prime}$ (comment les discours s'articulent entre eux). D'un autre côté, cette écoute polyphonique implique de ne pas croire que, quand un individu prend la parole dans un groupe, une seule personne parle : comme bakhtine l'a bien montré en remettant en question la croyance en l'unicité de l'auteur ${ }^{10}$. En effet, les paroles produites par un sujet n'ont pas qu'un seul auteur; dans le discours polyphonique, différents auteurs parlent en nous, dit bakhtine ${ }^{11}$. Les fonctions phoriques de porteparole, porte-symptôme, porte-affect, porte-haine décrites par René Kaës en sont des expressions.

Les chaînes associatives individuelles et groupales sont sous-tendues par une succession de ce que nous avons proposé de nommer " les scénarios de base » (Gimenez, 2000, 2006) que l'on peut repérer et transcrire. Il s'agit de scénarios énoncés dans le groupe et épurés des personnages et compléments qui qualifient et habillent le scénario. Ces scénarios sont structurés comme une phrase : sujet, verbe, complément sous la forme : "Quelqu'un fait quelque chose à quelqu'un d'autre, éventuellement devant un troisième qui regarde. » Ils ont la même structure que le fantasme ou le rêve, composés de trois places : celui qui agit, celui sur qui l'action est faite, celui qui regarde la scène. Le cœur du scénario en est le verbe, l'action. Comme chacun en a fait l'expérience, le passage du latent au manifeste implique souvent des modifications quant à ces places, dans des mouvements de réversibilité, inversion, substitution sujet-objet, actif-passif, déplacement en position d'observateur de la scène, etc.

Le premier temps dans l'observation de la production des discours du groupe consiste selon nous à repérer ces scénarios de base. Le second temps consiste à observer leur succession et leur articulation chez chaque sujet et dans le groupe, dans une séance et entre les séances. Le clinicien aura à rêver ces liens, et se demander en quoi ces scénarios de base figurent ou tentent de figurer ce qui se passe chez les sujets et dans le groupe. Les différentes places dans le scénario, traduisant des vertex différents mais complémentaires, pourront également être explorées : celle de l'acteur, celle de celui sur lequel on effectue l'action et celle de l'observateur. C'est ce qui est le plus souvent travaillé dans les groupes de jeux de rôle et possiblement dans les groupes de psychodrames.

Je prendrai ici l'exemple d'un workshop d'aetga, organisé à Aixen-Provence, quelques mois après le groupe de Marsala. Ce groupe est constitué de praticiens et d'analystes de groupes qui se soumettent à la même expérience transculturelle sans traducteur. Je suis frappé que le groupe commence sans que des règles de fonctionnement soient

9. R. Kaës, La parole et le lien. Processus associatifs dans les groupes, op. cit., p. 29.

10. Ibid., p. 35.

11. Ibid. 
énoncées. Ce qui me pose problème : et je me demande si l'on peut analyser ce qui se passe sans cadre clair et stable.

Les premières associations portent sur la langue que l'on va utiliser. Il est soutenu par plusieurs participants que ce n'est pas parce qu'on est en France pour cette rencontre qu'on va parler français. On exclut aussi très vite la langue allemande, ce qui fait émerger des remarques vives de plusieurs participants qui remarquent que cette langue a déjà été, à plusieurs reprises, mise de côté. On voit comment un premier scénario de base émerge : «On empêche quelqu'un de parler » ou plus précisément on empêche de parler la langue maternelle (le français, l'allemand).

Après quelques tours de paroles, une jeune femme proposera, pour mettre tout le monde d'accord (et éviter les affrontements), de parler anglais, pour que l'on se comprenne bien, et aussi soutient-elle (dans ce qui me semble être une rationalisation), pour aider la personne qui est chargée de prendre des notes. Ce que l'on peut traduire avec le scénario de base suivant: " On décide de ne parler qu'une seule langue (que tout le monde est censé connaître), une langue commune, l'anglais. " Nous ferons plus tard l'hypothèse que cette jeune femme portait (fonction phorique) un scénario et une stratégie du groupe visant à niveler les différences entre nous et ainsi les mouvements d'altérité et les angoisses qui pouvaient naître dans cette différence. Ceci justement à l'endroit où le dispositif a pour objet de travailler sur les différences culturelle et linguistique.

Dans le groupe, les participants, décident alors de parler « de ce qui fait lien entre nous, plutôt que de ce qui sépare ». Un participant évoque les deux parties d'un symbole que l'on peut remettre ensemble pour reconstruire l'unité originaire. On parle de ce qui est commun dans nos approches : la psychanalyse et la prise en compte de l'inconscient. Le troisième scénario de base pourrait ici être énoncé : "Les différences entre les membres du groupe sont gommées », ou « On retrouve l'unité en deçà de la séparation et des différences », ou encore : "On tait ce qui fait différence », ou enfin : «On ne parle que de ce qui relie ».

Mais les échanges sur ce qui est commun butent sur un élément de réalité : les participants font remarquer que nous n'avons pas tous la même formation, ni la même conception du groupe, ni les mêmes référents, ni les mêmes méthodes, ni les mêmes filiations théoriques. Un quatrième maillon de la chaîne associative est ainsi constitué par des échanges sur les conceptions du groupe par chacun et pourrait ainsi s'énoncer : "Chacun est différent. » Cet échange se cristallise autour d'un événement particulier qui a eu lieu dans le grand groupe à Marsala : un participant avait déplacé sa chaise, pour l'amener presque au centre du groupe, et ainsi mobilisé une immense tension chez les cent cinquante participants. Quelques conducteurs du grand groupe on fait de même, s'inscrivant dans la dynamique de ce qui émergeait. D'autres conducteurs de groupes n'ont pas bougé, car, pour eux, il s'agissait 
d'un acting du participant suivi par des conducteurs de groupe peu rigoureux sur le cadre.

Des tensions émergent alors dans notre groupe aixois, chacun fait une lecture personnelle de cet événement, personne n'est d'accord sur les détails, on revient sur ce qui s'est vraiment passé, sur les faits, sur ce qu'on a ressenti, sur les hypothèses que chacun a émises. Les liens entre les participants se tendent. Le groupe se divise en deux sousgroupes. Ce qui semblait sous-tendre l'ensemble des échanges émerge enfin : « Notre différence nous fait violence. »

Le sixième scénario de base de cette chaine associative pourrait ainsi être énoncé : «On regarde (et assume) l'effet de violence que produisent nos différences ». Le groupe reste un moment sur cette question difficile et douloureuse de la violence de l'interculturel, la place de l'étranger (l'autre comme étranger mais aussi l'étranger en soi) et arrive à ce constat que, pour travailler ensemble, nous devons préalablement reconnaître cette violence fondamentale fondée sur nos différences. On parle alors de la difficulté à se comprendre, du temps trop long qu'on passe pour saisir ce que veulent dire certains, et que cela est quelquefois tout à fait intolérable. en écho, un participant repère qu'il a essayé tout au long de la journée de proposer des thèmes pour faire " lien groupal », et éviter cette confrontation trop difficile, mais en vain. Au bout d'un moment, le regard est porté sur cette violence qui traverse chacun, et la tension commence à baisser. Finalement, un membre du groupe dit : «C'est mon problème de ne pas comprendre, je peux poser des questions. \"

Les mouvements projectifs du groupe semblent apaisés et chacun parvient alors à regarder en lui-même, et parler de ce qui, de la différence de l'autre, mobilise son narcissisme, ses blessures narcissiques et sa colère... mais aussi, en fond, de la peine d'être seul en présence des autres tellement différents... Nous pourrons alors commencer à parler de ce qui a amené à ne pas énoncer les règles du cadre de ce groupe en début de notre séance : en écho au groupe de Marsala, comme pour ne pas faire émerger trop vite nos différences... Nous voyons bien, dans cette séquence clinique, comment les trois premiers niveaux d'observation s'articulent, dans un mouvement de recul, de décalage, de " prise de hauteur » sur le sensoriel, le matériel clinique interne et le repérage de la chaîne associative groupale. Advient un espace pour l'observation de l'observation elle-même, qui pourra laisser advenir la pensée, et le travail de l'hypothèse.

\section{LES HYPOTHESES SUR LE PROCESSUS REPERE : LA FONCTION META (TIERCEISATION)}

Le quatrième niveau d'observation correspond ainsi au quatrième niveau de réalité psychique du groupe : il s'agit de l'observation de l'observation elle-même effectuée dans les niveaux 1, 2 et 3. Quand 
nous conduisons un groupe, nous faisons l'expérience qu'une partie de nous est pleinement dans le groupe pendant qu'une autre partie de nous auto-observe ce qui se passe : elle observe notre façon d'observer, mais aussi ce que nous pensons, ce que nous nous disons, comment nous sélectionnons nos perceptions, les traitons, les transformons... L'attention est ici focalisée sur l'observateur que nous sommes et sur son activité d'observation. Il s'agit de la position dite « méta » spécifique à l'observation clinique : «Je m'observe en train d'observer et je peux commenter ce qui est repéré avec recul et décalage. » Cette métaobservation est le soubassement de notre discussion interne pendant la séance, avec les modèles de référence que nous utilisons, ce qui rend possible le travail de l'hypothèse sur les processus repérés. Dans la prise de notes, ces éléments sont consignés dans la quatrième colonne en regard des colonnes 1,2 , et 3 correspondantes.

L'observation de l'observation est rendue possible par la capacité à être non seulement pleinement présent dans la situation (premier et second niveaux de réalité psychique du groupe) mais aussi dans une " position méta », à côté, décalée, observateur et commentateur de ce qui se passe.

\section{Dissociation et fonction méta}

La «position méta » est sous-tendue par une forme spécifique de dissociation. Janet (1989) a décrit très précisément le mécanisme dissociatif, souvent présent après des expériences traumatiques et dans les états modifiés de conscience. Je propose de nommer " dissociation fonctionnelle ", une forme non pathologique de la dissociation permettant de rendre quasi-autonomes plusieurs parties du psychisme. C'est ce qui se passe, quand je conduis : je suis totalement présent à la conduite de mon véhicule et, en même temps, une autre part de moi peut développer une rêverie sur ce que je vais dire lors d'une future intervention. Si un obstacle surgit sur la route, je suis tout à fait disponible pour réagir de façon adaptée.

Dans l'activité d'observation clinique, ces deux parts de soi sont à la fois séparées et en dialogue : la première expérimente et éprouve ce qui est en train de se passer, alors que la seconde, simultanément, observe l'observateur et commente ce qui se passe. Un tel phénomène a été décrit par P. Casement ${ }^{12}$ dans cette position subjective qu'il nomme le « superviseur interne ». Le fonctionnement du superviseur interne est sous-tendu par une approche et une écoute bifocale de la situation clinique : une part du clinicien est engagée dans la rencontre alors qu'une autre part de lui est observateur et analyste ce qui se passe grâce à ce qu'il a nommé la "dissociation thérapeutique ». Casement va un peu plus loin et nomme «identification d'épreuve » l'utilisation simultanée 
par le clinicien de « deux points de vue [...], celui du patient et le sien propre, un peu comme on [écoute] différentes voix dans la musique polyphonique ${ }^{13} »$.

\section{Identification d'épreuve et écoute polyphonique}

Nous voyons ici comment cet auteur essaie d'aborder un type d'observation et d'écoute qu'il conviendrait de nommer à partir des travaux de René Kaës (1994) «l'observation polyphonique du groupe ». Dans la position méta, se développe la capacité à explorer et maintenir coprésents sans conflit, et en dialogue, plusieurs vertex (plusieurs points de vue) : celui du ou des clinicien(s) (coconducteurs du groupe), et celui (ou ceux) des participants. Ce dialogue interne chez le clinicien pourrait être comparé à une sorte d'intervision, en temps réel, entre plusieurs parties de son groupe interne.

\section{Ce que « méta » veut dire}

En grec, le préfixe « méta » exprime, tout à la fois, la réflexion, le changement, la succession, le fait d'aller au-delà, à côté de, entre ou avec. La position méta nécessite de " sortir de soi ", de se décaler, de se dé-prendre de son point de vue, de ses propres représentations saturantes, de prendre du recul par rapport à notre cadre de référence, dans une rêverie, où l'on peut jouer (au sens winnicottien) à se regarder du dehors ou d'un autre point de vue... comme en observant un autre. Dit autrement, il s'agit d'une certaine manière d'être médiateur de soi-même sur le regard que nous portons sur nous... Par cette prise de dis-tance, un espace psychique s'ouvre entre la partie du sujet qui observe (auto-observe) et une autre qui est observée. Chez le sujet auto-obser-vant peut alors se développer une pensée autoréflexive. Le sujet va développer une auto-représentation de lui-même et de son activité psy-chique, de ses mouvements internes, et de son activité d'observation. La position méta est en lien avec la capacité à développer « le souci de soi », comme le nomme Michel Foucault : s'observer, s'accompagner, prendre soin, envelopper et contenir...

\section{De l'introjection et des identifications}

La position méta naît de l'introjection du regard de l'autre (fonction miroir) posée sur soi-même, elle devient auto- et méta-regard, porté réflexivement sur soi-même. La position méta est également soutenue par la capacité à s'identifier au regard d'un autre [pour soi], et de " plus d'un autre ». Ce qui permet de s'appréhender « soi-même comme un autre », selon la belle expression de Ricœur (1990). 


\section{Méta et théorisation flottante}

La position méta favorise l'activité de " théorisation flottante ${ }^{14}$ » et le «travail de l'hypothèse ", c'est-à-dire la compréhension de la situation clinique, par le repérage et la mise en lien d'indices cliniques convergents. La théorisation flottante a une fonction pare-excitative (et non défensive), de filtre de la violence des situations cliniques, mais aussi de transformation élaborative. Ce quatrième niveau de réalité psychique du groupe convoque, réveille et excite la pulsion épistémophilique (lien K knowledge ou C connaissance, selon bion, 1962). Il s'agirait ici d'un développement épanouissant d'un équivalent de la pulsion épistémophilique du côté de l'être : le mouvement qui nous pousse, chacun, à nous connaître mieux, plus intimement, tel que peut le révéler le partage avec un groupe, «par le travail de l'intersubjectivité » (Kaës, 2002).

\section{aetga}

Dans le matériel clinique présenté, nous avons pu repérer comment l'élaboration du matériel clinique se fait en regard avec la capacité des cliniciens et du groupe à penser ce qui se passe, c'est-à-dire à émettre des hypothèses qui rendent compte des phénomènes repérés et de la succession des scénarios de base constitutifs de la chaîne associative du groupe : " On empêche quelqu'un de parler (une langue étrangère) »; " On décide de ne parler qu'une seule langue (commune et ainsi gommer les différences et l'altérité) »; " On tait ce qui fait différence »; puis « On commence à repérer les différences de chacun »; pour enfin parvenir à regarder ce qui sous-tendait cette succession associative : « Notre différence nous fait violence » (c'est pour cela qu'on s'en protège); ce qui aboutit à « On regarde (et assume) l'effet de violence que produisent nos différences ». Ce groupe actualise ce que René Kaës (1998) a décrit comme « la troisième différence » qu'il ajoute à la différence des sexes et la différence des générations. Cette troisième différence est celle de la culture, décrite et mise en évidence à partir de ces groupes transculturels. Les trois grandes formes de différences s'organisent autour de trois couples d'oppositions : l'opposition humain/non humain, dont la transgression est de l'ordre du monstrueux ; l'opposition humain/humain dans l'ordre de la différence des générations et des sexes, et dont la transgression produit la catégorie de l'incestueux ; l'opposition humain/humain dans l'ordre des appartenances sociales et de la culture, " la transgression de cette opposition produit la catégorie de la subversion et de l'ennemi ${ }^{15} \gg$.

14. P. Aulagnier citée par J. McDougall, Théâtre du Je (1982), Paris, Gallimard, 2004, p. 21. 15. R. Kaës, « Différence culturelle, souffrance de la langue et travail du préconscient dans deux dispositifs de groupe ", dans Différence culturelle et souffrances de l'identité, Paris, Dunod, 1998, p. 20. 
René Kaës soutient que cette " troisième différence ", la différence culturelle, «métaphorise toutes les autres » et que les termes de la transgression sont interchangeables : l'autre culturel, l'étranger, est un animal (un sous-homme), un monstre, un enfant, une femme, un malade, un sauvage, etc.

« Autrement dit, la différence culturelle peut être le déplacement ou la condensation de la différence sexuelle ou la différence entre les générations, ou des deux à la fois ${ }^{16}$. "

Nous avons observé ces différences dans les quatre niveaux de réalité psychique des groupes : au niveau sensoriel (dans l'expérience de la babélisation, du chaos et de la cacophonie) ; au niveau contretransférentiel et intertransférentiel (dans le repérage de la violence émotionnelle fondamentale produite par situation de mise en groupe, se réfléchissant dans notre groupe interne) ; au niveau de la chaîne associative groupale et la succession des scénarios de base, comme traduction de plus en plus proche de ce qui se passe au niveau du groupe, face à la violence de l'altérité radicale ; au niveau de la position méta et du travail de l'hypothèse comme la méta-observation et mise en forme de ce qui, en nous, demeure radicalement étranger, inconnu, non conscient, non accessible et incompréhensible (en un mot : non moi).

\section{BIBLIOGRAPHIE}

bion, w.r. 1962. Aux sources de l'expérience, Paris, Puf, 1979.

bion, w.r. 1967. Réflexion faite, Paris, Puf, 1983.

bion, w.r. 1970. L'attention et l'interprétation, Paris, Payot, 1974.

bote11a, c. ; bote11a, s. 1989. « La problématique de la régression formelle de la pensée et de l'hallucinatoire », Revue française de psychanalyse, Colloque SPP Unesco du 14 et 15 janvier 1989, p. 63-90.

casement, P. 1985. À l'écoute du patient, Paris, Puf.

freUd, s. 1910. «Perspectives d'avenir de la thérapeutique analytique », dans $L a$ technique psychanalytique, Paris, Puf, p. 23-34.

gimenez, g. 2000. Clinique de l'hallucination psychotique, Paris, Dunod.

gimenez, g. 2006. « La construction d'une chaîne associative groupale dans le travail psychanalytique avec les patients psychotiques en groupe : du chaos dissociant à une possible polyphonie », Revue de psychothérapie psychanalytique de groupes, $\mathrm{n}^{\circ} 47$, p. 79-91.

gimenez, g. ; barthélémy, S. 2011. «Le temps de la prise de notes. Propositions d'une méthode de notations dans les groupes cliniques », Revue de psychothérapie psychanalytique de groupes, $\mathrm{n}^{\circ}$ 56, p. 171-185.

grotstein, J. 1981. «Primal splitting, the background Object of Primary Identification, and Others Self-Objects », dans Splitting and Projective Identification, New York, Jason Aronson ed., p. 77-89. 
Janet, P. 1889. L'automatisme psychologique. Essai de psychologie expérimentale sur les formes inférieures de l'activité humaine, Paris, L'Harmattan.

Kaës, R. 1976. « L'analyse inter-transférentielle », dans Kaës R., Anzieu D. et coll., Désir de former et formation du savoir, Paris, Dunod.

Kaës, R. 1982. «L'inter-transfert et l'interprétation dans le travail psychanalytique groupal », dans R. Kaës, A. Missenard, J.-C. Ginoux et coll., Le travaii psychanalytique dans les groupes. 2, Les voies de l'élaboration, Paris, Dunod.

Kaës, R. 1994. La parole et le lien. Processus associatifs dans les groupes, Paris, Dunod.

Kaës, R. 1998. " Différence culturelle, souffrance de la langue et travail du préconscient dans deux dispositifs de groupe ", dans Différence culturelle el souffrances de l'identité, Paris, Dunod, p. 45-87.

Kaës, R. 2002. "Les groupes de formation : perspective psychanalytique », Le Journal des psychologues, $\mathrm{n}^{\circ} 203$, p. 22-25.

K1ein, m. 1946. " Notes sur quelques mécanismes schizoïdes », dans M. Klein, P. Heimann, S. Isaac, J. Riviere, Développements de la psychanalyse, Paris, Puf, 1966, p. 274-300.

mcdouga11, J. 1982. Théâtre du Je, Paris, Gallimard, 2004.

ricour, p. 1990. Soi-même comme un autre, Paris, Le Seuil.

Searles, 1979. Le contre-transfert, Paris, Gallimard, 1981.

Winnicott, D.W. 1974. "La crainte de l'effondrement », Nouvelle revue de psychanalyse, $\mathrm{n}^{\circ} 11,1975$, p. 35-44. 